ИЗВЕСТИЯ АКАДЕМИИ НАУК ЭСТОНСКОИ ССР. ФИЗИКА * МАТЕМАТИКА PROCEEDINGS OF THE ACADEMY OF SCIENCES OF THE ESTONIAN SSR. PHYSICS * MATHEMATICS

$1986,35,4$

УДК $535.338 .32: 535.338 .334+531.761$

V. HIZHNYAKOV, Inna REBANE

\title{
LINE NARROWING IN TIME-DEPENDENT SPECTRA
}

\section{Introduction}

An increase of the time interval $t$ between excitation and recording may lead to the narrowing of spectral lines in time-dependent spectra (see, e. g. papers $\left[{ }^{1-7}\right]$ on the Mössbauer effect and $\left[{ }^{8-12}\right]$ on optical spectra). In the obtaining of narrow lines in these spectra the choice of spectral resolution $\eta$ is of considerable importance: the narrowest lines are not obtained at $\eta=0$, as it is in the stationary case, but at $\eta>0$. Particularly, in a time-dependent spectrum of resonant fluorescence (luminescence), the linewidth depends on $|\gamma-\eta|$, in time-dependent spectra of absorption and scattering, however, it is determined respectively by the parameters $\left|\gamma-\eta_{0}\right|$ and $\left|\eta-\eta_{0}\right| \quad\left(\gamma\right.$ is the natural linewidth and $\eta_{0}$, the spectral linewidth (reverse duration) of excitation). As a result, the lines with an uncertainty-principle-limited halfwidth $t^{-1}$ can be observed at $\eta=\gamma$ (fluorescence), $\eta_{0}=\gamma$ (absorption), and $\eta=\eta_{0}$ (scattering); in these cases $\eta, \eta_{0}$ and $\gamma$ are compensated.

The compensation effect for luminescence and scattering has been considered in $\left[{ }^{8,9}\right]$ within the frames of the quantum theory of resonant secondary radiation (RSR). In $\left[{ }^{13}\right]$ this effect was also obtained for luminescence (modulational broadening excluded). The compensation of the reverse duration of the exciting pulse $\eta_{0}$ and natural linewidth $\gamma$ in a time-dependent absorption spectrum has been obtained in $\left.{ }^{[10-12}\right]$. Note also papers $\left[{ }^{1,2}\right]$ which showed that in the time-dependent spectra of the Mössbauer $\gamma$ quanta passing through the resonant absorber, a minimum with an uncertainty-principle-limited halfwidth $t^{-1}$ can be observed. Here, as will be shown below, a compensation of the natural linewidth of the source and resonance absorbed takes place.

In this communication, the compensation effect in time-dependent RSR spectra is studied with regard to the homogeneous (modulational, $\left.\gamma_{1}\right)$ and inhomogeneous $\left(\gamma_{2}\right)$ line broadening. The consideration is based on a simple classical radiation theory. It is shown that on a proper choice of $\eta$ and $\eta_{0}$, and at small $\gamma_{1}$ and $t^{-1}$ the time-dependent RSR spectra may reveal lines with the halfwidth considerably less than $\eta$, $\eta_{0}$, $\gamma$ and $\gamma_{2}$. In particular, in the case of a large inhomogeneous or Doppler broadening in this spectrum at $\gamma_{1}=0$, one can also obtain a line with an uncertainty-principle-limited halfwidth $t^{-1}$. This allows one to use systems with a large (as compared to $\gamma$ ) inhomogeneous and Doppler broadening of spectral lines to observe the compensation effect.

\section{2.. Time-dependent spectrum}

Let us determine a time-dependent radiation spectrum as the counting rate of the photons passed through the spectrometer with mean frequ- 
ency $\Omega$ and spectral resolution $\eta$ at time moment $t$. The photons are registered by a photodetector with a broad sensitivity band. In this case $\left[{ }^{14,15,8}\right]$,

$$
I \sim|E(t)|^{2},
$$

where $E(t) \equiv E(t, \Omega, \eta)$ is the field intensity of the light wave passing through the spectrometer with the average transmission frequency $\Omega$ and spectral resolution $\eta$.

Let us regard the influence of the spectrometer on radiation. First, the signal is delayed, which is connected with the finiteness of the flight of photons through the spectrometer. In general this time depends on the frequency of the light $\Omega$, which leads to an increase of the duration of the passed-through light pulse. However, in subtractive dispersive spectrometers, the dependence on $\Omega$ is eliminated by the compensation of the path difference and the increase of signal duration mentioned above, is absent $\left[{ }^{16}\right]$. In this case, the time of flight of photons through the spectrometer can be subtracted from the observation time.

Second, the spectrometer changes the form of the passed-through signal as a result of wave filtration over the spectrum. The latter is described by the formula

$$
E(\omega)=E^{\prime}(\omega) C(\omega),
$$

where $E^{\prime}(\omega)$ and $E(\omega)$ are the Fourier components of the electric field intensity for the incident $\left(E^{\prime}\right)$ radiation and for the one passed-through (E) the spectrometer; $|C(\omega)|^{2}$ is the function of spectrometer filtration determining the law of transforming the intensities of monochromatic waves by the spectrometer. It follows from formula (2) that the incident and passed-through fields are connected by the integral relation

$$
E(t)=\frac{1}{2 \pi} \int_{-\infty}^{\infty} \mathrm{dt}_{1} E^{\prime}\left(t_{1}\right) C\left(t-t_{1}\right),
$$

where

$$
C(t)=\int_{-\infty}^{\infty} d \omega e^{i \omega t} C(\omega)
$$

is the spectrometer transmission function. This function (as well as $C(\omega)$ ) depends parametrically on the mean frequency of the spectrometer transmission $\Omega$ and on the spectral resolution $\eta$.

The main property of the function $C(t)$ adhering to the causality principle is that it is zero at $t<0$ :

$$
C(t)=\theta(t) C(t)
$$

$(\theta(t)$ is the Heaviside stepwise function: $\theta(t)=0$ if $t<0, \theta(t)=1$ if $t \geqslant 0)$. Consequently, its Fourier transform $C(\omega)$ can have poles only in the upper half of the complex plane. Particularly, this property is satisfied for the function

$$
C(\omega)=i C_{0}(\Omega-\omega+i \eta)^{-1}
$$

( $C_{0}$ is a constant) describing the Fabri-Perrot etalon [ $\left.{ }^{17}\right]$. In this case, the filtration function $|C(\omega)|^{2}$ is Lorentzian, while the transmission one, $C(t)$, is exponential:

$$
C(t)=2 \pi \theta(t) C_{0} e^{i \Omega t-\eta t} .
$$


Formula (3), with the allowance (5) made for $\hat{C}(t)$, gives

$$
E(t)=\frac{1}{2 \pi} \int_{-\infty}^{t} \mathrm{dt}_{1} E^{\prime}\left(t_{1}\right) C\left(t-t_{1}\right) .
$$

In particular, in the case of a Lorentzian filtration

$$
E(t)=C_{0} \int_{-\infty}^{t} \mathrm{dt}_{1} e^{i \Omega\left(t-t_{1}\right)-\eta\left(t-t_{1}\right)} E^{\prime}\left(t_{1}\right) .
$$

It follows from these formulae that the spectrometer integrates signals with various delay $t-t_{1}$ (in the limits $\sim \eta^{-1}$ ). Physically this corresponds to a signal interference with various $t-t_{1}$. As will be shown below, it is this interference that leads to the compensation effect.

\section{Resonance fluorescence}

Let us regard a classical electric field of the radiation emitted by a single atom (nucleus) after its instant excitation. This field, uvidently, has the form

$$
E^{\prime}\left(t_{1}\right)=E_{0} \theta\left(t_{1}\right) e^{i \omega_{0} t_{1}-\gamma t_{1}},
$$

where $E_{0}$ is the amplitude and $\omega_{0}$, the frequency of the electronic transition. A substitution of formula (9) into (8) gives

$$
E(t)=E_{0} C_{0} e^{i \omega_{0} t-\gamma t} \frac{e^{i x t-\overline{\gamma t}}-1}{\bar{\gamma}+i x},
$$

where $x=\Omega-\omega_{0}, \bar{\gamma}=\gamma-\eta$. Therefore; the time-dependent radiation (fluorescence) spectrum is equal to $\left[{ }^{8,13}\right]$

$$
I \sim \frac{e^{-2 \gamma t}+e^{-2 \eta t}-2 e^{-(\gamma+\eta) t} \cos x t}{x^{2}+\bar{\gamma}^{2}} .
$$

In formula (11), the luminescence line broadening due to the processes of transverse (phase) relaxation modulating the transition frequency $\omega_{0}$, is not taken into account. In the present case of momentary excitation, these processes can be taken into account by integrating formula (11) over $\omega_{0}$ with a proper distribution function $\varrho\left(\omega_{0}\right)$. If $\varrho\left(\omega_{0}\right)$ is the Lorentzian function with the maximum $\bar{\omega}_{0}$ and the halfwidth $\gamma_{1}$, then ${ }^{*}\left[{ }^{8}\right]$

$$
\begin{gathered}
I \sim e^{-2 \gamma t} \frac{\Gamma / \bar{\gamma}}{\bar{x}^{2}+\Gamma^{2}}+e^{-2 \eta t} \frac{\Gamma_{1} / \bar{\gamma}}{\bar{x}^{2}+\Gamma_{1}^{2}}- \\
-2 e^{-\left(\gamma+\eta+\gamma_{1}\right) t} \frac{\left(\bar{x}^{2}+\Gamma \Gamma_{1}\right) \cos \bar{x} t+2 \gamma_{1} \bar{x} \sin \bar{x} t}{\left(\bar{x}^{2}+\Gamma^{2}\right)\left(\bar{x}^{2}+\Gamma_{1}^{2}\right)}
\end{gathered}
$$

Formula (12) can also be obtained from formulae (21)-(23) when it is considered that on momentary excitation $E_{1}\left(t_{2}\right) \sim \delta\left(t_{2}\right)$. 


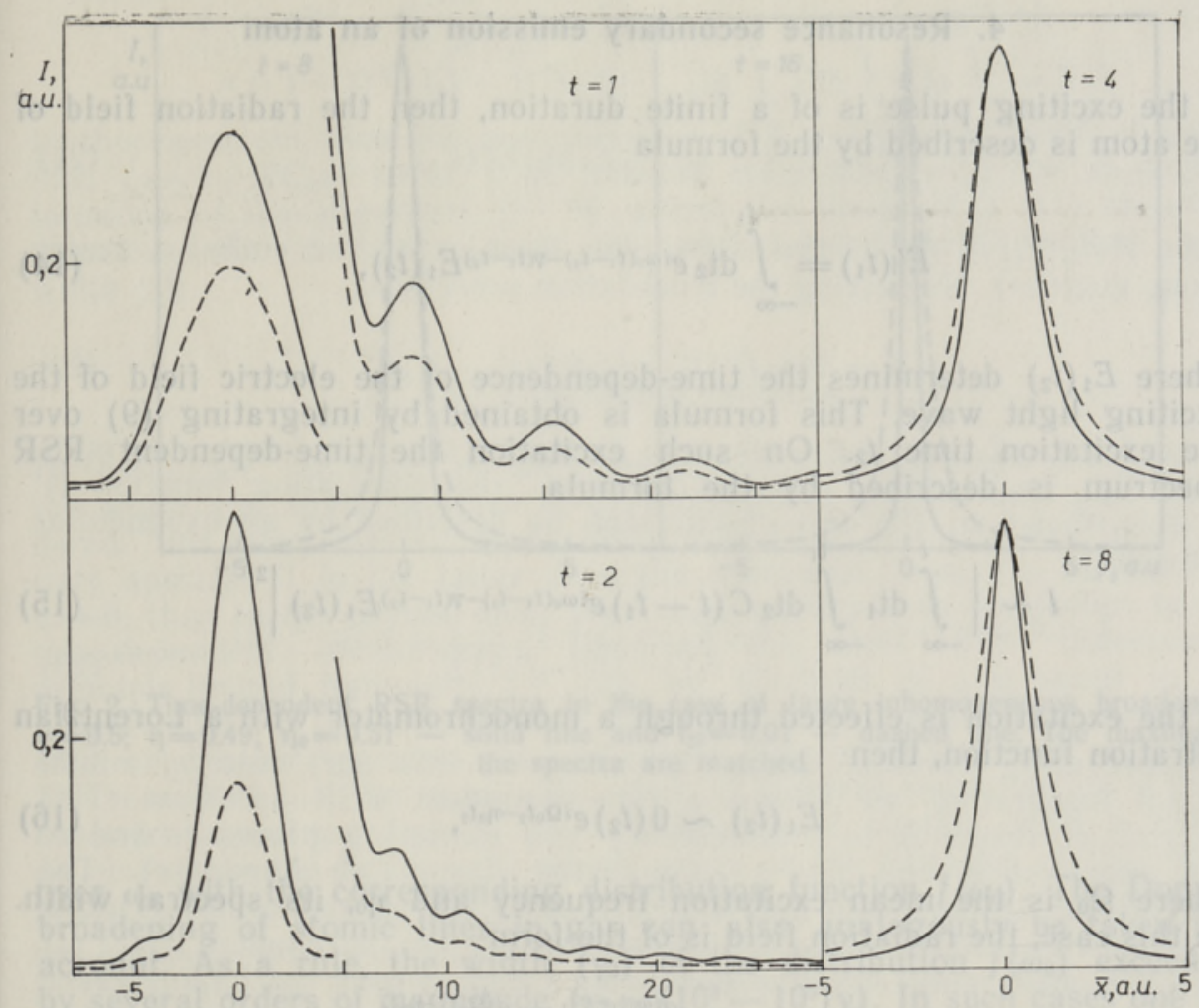

Fig. 1. Time-dependent resonance fluorescence spectra with account of dephasing (modulational) broadening: $\gamma_{1}=\gamma=0.5 ; \eta=0.49-$ solid line and $\eta=0.01-$ dashed line. For $t=4.8$ the maxima of the spectra are matched.

where $\bar{x}=\Omega-\overline{\omega_{0}}, \Gamma=\bar{\gamma}-\gamma_{1}, \Gamma_{1}=\bar{\gamma}+\gamma_{1}$. According to formulae (11) and (12) (see also Fig. 1) the line under consideration has an oscillating form, whereas the width of the main (central) maximum depends not on the sum of $\gamma$ and $\eta$ (as in the case of stationary spectra) but on their difference. Consequently, in this spectrum the effect of compensating the decay width and spectrometer resolution takes place. The narrowest line is obtained in the case of $\eta=\gamma$, when the line halfwidth is determined by $\gamma_{1}$ and $t^{-1}$ :

$$
\begin{gathered}
I \sim \frac{\mathrm{e}^{-2 \gamma t}}{\bar{x}^{2}+\gamma_{1}^{2}}\left[1+\gamma_{1} t-e^{-\gamma_{1} t} \cos \bar{x} t-\right. \\
\left.-\frac{2 \gamma_{1}^{2}}{\bar{x}^{2}+\gamma_{1}^{2}}\left(1-e^{-\gamma_{1} t} \cos \bar{x} t+\frac{\bar{x}}{\gamma_{1}} e^{-\gamma_{1} t} \sin \bar{x} t\right)\right] .
\end{gathered}
$$

Consequently, in time-dependent luminescence spectra the natural linewidth $\gamma$ can be compensated by spectral resolution $\eta$ but the modulational broadening $\gamma_{1}$ cannot. If $\eta=\gamma$ and $\gamma_{1}=0$ then $I \sim e^{-2 \gamma t}(1-\cos \bar{x} t) / \bar{x}^{2}$ which is the line with uncertainty-principle-limited halfwidth $t^{-1}$. 


\section{Resonance secondary emission of an atom}

If the exciting pulse is of a finite duration, then the radiation field of the atom is described by the formula

$$
E^{\prime}\left(t_{1}\right)=\int_{-\infty}^{t_{1}} \mathrm{dt}_{2} e^{i \omega_{0}\left(t_{1}-t_{2}\right)-\gamma\left(t_{1}-t_{2}\right)} E_{1}\left(t_{2}\right),
$$

where $E_{1}\left(t_{2}\right)$ determines the time-dependence of the electric field of the exciting light wave. This formula is obtained by integrating (9) over the excitation time $t_{2}$. On such excitation the time-dependent RSR spectrum is described by the formula

$$
I \sim\left|\int_{-\infty}^{t} \mathrm{dt}_{1} \int_{-\infty}^{t_{1}} \mathrm{dt}_{2} C\left(t-t_{1}\right) e^{i \omega_{0}\left(t_{1}-t_{2}\right)-\gamma\left(t_{1}-t_{2}\right)} E_{1}\left(t_{2}\right)\right|^{2} .
$$

If the excitation is effected through a monochromator with a Lorentzian filtration function, then

$$
E_{1}\left(t_{2}\right) \sim \theta\left(t_{2}\right) e^{i \Omega_{0} t_{2}-\eta_{0} t_{2}},
$$

where $\Omega_{0}$ is the mean excitation frequency and $\eta_{0}$, its spectral width. In this case, the radiation field is of the form

$$
E^{\prime}\left(t_{1}\right) \sim \theta\left(t_{1}\right) \frac{e^{i \omega_{0} t_{1}-\gamma t_{1}}-e^{i \Omega_{0} t_{1}-\eta_{0} t_{1}}}{\eta_{0}-\gamma+i\left(\omega_{0}-\Omega_{0}\right)} .
$$

Substituting this formula into (8), after integrating and taking square by modulus, we obtain

$$
\begin{gathered}
I \sim \frac{e^{-2 \eta t}}{x_{0}^{2}+\gamma_{0}^{2}}\left[\frac{1+e^{-2 \bar{\gamma} t}-2 e^{-\overline{\gamma t}} \cos x t}{x^{2}+\bar{\gamma}^{2}}+\frac{1+e^{2 \eta \eta t}-2 \overline{e^{\bar{\eta} t}} \cos y t}{y^{2}+\bar{\eta}^{2}}-\right. \\
\left.-2 \operatorname{Re} \frac{\left(1-e^{i x t-\bar{\gamma} t}\right)\left(1-e^{i y t+\bar{\eta} t}\right)}{(x+\overline{i \gamma})(y-\overline{i \eta})}\right],
\end{gathered}
$$

where $x_{0}=\Omega_{0}-\omega_{0}, y=\Omega_{0}-\Omega, \gamma_{0}=\gamma-\eta_{0}, \bar{\eta}=\eta-\eta_{0}$. In this formula, the first term in wavy brackets describes the luminescence-type RSR, the second one, the scattering-type RSR and the third one, their interference. The luminescence linewidth is determined by the parameters $|\bar{\gamma}|=|\gamma-\eta|$ and $t^{-1}$, the scattering linewidth, by $|\bar{\eta}|=\left|\eta-\eta_{0}\right|$ and $t^{-1}$. Consequently, in the luminescence line, $\eta$ and $\gamma$ are compensated, in the scattering line, however, $\eta$ and $\eta_{0}$.

\section{Compensation effect for inhomogeneously broadened lines}

In impurity crystals at low temperatures purely electronic lines are inhomogeneously broadened. The influence of the broadening on the time-dependent RSR spectrum can be found by integrating formula (18) 


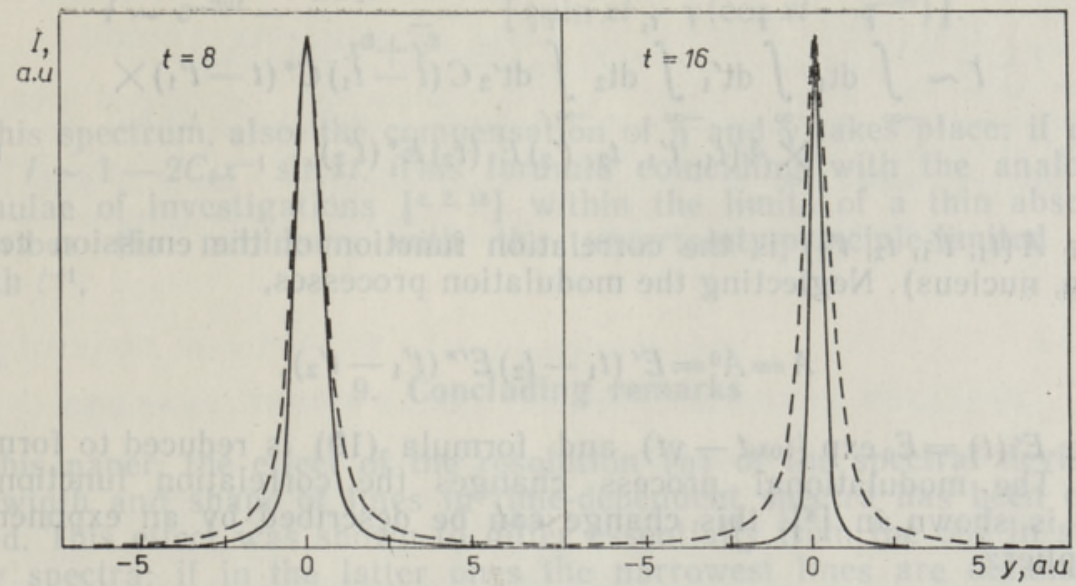

Fig. 2. Time-dependent RSR spectra in the case of large inhomogeneous broadening: $\gamma=0.5 ; \eta=0.49 ; \eta_{0}=0.51$ - solid line and $\eta_{0}=0.01-$ dashed line. The maxima of the spectra are matched.

over $\omega_{0}$ with the corresponding distribution function $f\left(\omega_{0}\right)$. The Doppler broadening of atomic lines in gas can also analogously be taken into account. As a rule, the width $\left(\gamma_{2}\right)$ of the distribution $f\left(\omega_{0}\right)$ exceeds $\gamma$ by several orders of magnitude $\left(\gamma_{2} \sim\left(10^{3}-10^{5}\right) \gamma\right)$. In such cases one can take $f\left(\omega_{0}\right)=$ const. In this approximation,

$I \sim e^{-\left(\eta_{0}+\eta\right) t}\left[\frac{\bar{\gamma} e^{\bar{\eta} t}+\gamma_{0} e^{-\bar{\eta} t}-\Delta e^{-\Delta t}}{4 \gamma_{0} \bar{\gamma}\left(y^{2}+\bar{\eta}^{2}\right)}+\frac{\Delta\left(e^{-\Delta t}-\cos y t\right)-y \sin y t}{\left(y^{2}+\overline{\eta^{2}}\right)\left(y^{2}+\Delta^{2}\right)}\right]$,

where $\Delta=2 \gamma-\eta_{0}-\eta$. We can see that the form of the line under consideration is determined by the parameters $\gamma-\eta, \eta-\eta_{0}$ and $t^{-1}$. It follows that the compensation effect in the time-dependent RSR spectrum can be observed also for inhomogeneously broadened luminescence lines (see Fig. 2). For example, if $\left|\eta-\eta_{0}\right|=\varepsilon, \quad\left|\gamma-\eta_{0}\right|=|\eta-\gamma|=\varepsilon / 2$, then the RSR spectral line has the form

$$
I \sim \frac{e^{-\left(\eta_{0}+\eta\right) t}}{\varepsilon\left(y^{2}+\varepsilon^{2}\right)}\left(e^{e t}-e^{-\varepsilon t}-2 \varepsilon y^{-1} \sin y t\right) .
$$

Within the limit $\varepsilon \rightarrow 0$ we have $I \sim y^{-2} e^{-2 \gamma t}\left(t-y^{-1} \sin y t\right)$, which is the line with the uncertainty-principle-limited halfwidth $t^{-1}$.

\section{RSR with regard to phase relaxation}

Let us now take into account the phase relaxation in RSR, which modulates $\omega_{0}$. In the present case of a finite-duration excitation, the corresponding processes of relaxation and excitation overlap in time, which necessitates their simultaneous consideration. The latter is possible by using the RSR correlation function formalism [ $\left.{ }^{8}\right]$. For this purpose let us present the photon counting rate in the form 


$$
\begin{gathered}
I \sim \int_{-\infty}^{t} \mathrm{dt}_{1} \int_{-\infty}^{t} \mathrm{dt}^{\prime}{ }_{1} \int_{-\infty}^{t_{1}} \mathrm{dt}_{2} \int_{-\infty}^{t_{1}} \mathrm{dt}_{2}{ }_{2} C\left(t-t_{1}\right) C^{*}\left(t-t^{\prime}{ }_{1}\right) \times \\
\times A\left(t_{1}, t^{\prime}{ }_{1}, t_{2}, t^{\prime}{ }_{2}\right) E_{1}\left(t_{2}\right) E_{1}^{*}\left(t_{2}^{\prime}\right),
\end{gathered}
$$

where $A\left(t_{1}, t^{\prime}{ }_{1}, t_{2}, t_{2}^{\prime}\right)$ is the correlation function of the emission centre (atom, nucleus). Neglecting the modulation processes,

$$
A=A^{0}=E^{\prime}\left(t_{1}-t_{2}\right) E^{\prime *}\left(t_{1}^{\prime}-t_{2}^{\prime}\right),
$$

where $E^{\prime}(t)=E_{0} \exp \left(i \omega_{0} t-\gamma t\right)$ and formula (19) is reduced to formula (14). The modulational process changes the correlation function $A$. As it is shown in $\left[^{8}\right]$, this change can be described by an exponential multiplier:

$$
\begin{gathered}
A=A^{0} \exp \left[-\gamma_{1}\left(t_{1}-t_{2}+t^{\prime}{ }_{1}-t_{2}^{\prime}+\left|t_{1}-t^{\prime}{ }_{1}\right|+\left|t_{2}-t^{\prime}{ }_{2}\right|-\right.\right. \\
\left.\left.-\left|t_{1}^{\prime}-t_{2}\right|-\left|t_{1}-t^{\prime}{ }_{2}\right|\right)\right] .
\end{gathered}
$$

By substituting formulae (22), (23) and (17) into (21), and by a subsequent integrating we obtain a time-dependent RSR spectrum. This spectrum corresponds to luminescence and scattering processes and to their interference, and has a complicated form (see Appendix). The shape of the luminescence line is described by the parameters $|\gamma-\eta|, \gamma_{1}$ and $t^{-1}$, while that of the scattering line, by the parameters $\left|\eta-\eta_{0}\right|$ and $t^{-1}$. Consequently, the phase relaxation causes only the luminescence line broadening and only this broadening cannot be compensated either by $\eta$ or $\eta_{0}$.

\section{Time-dependent absorption spectrum}

The time-dependent absorption spectrum is determined by the counting rate of the emitted (scattered) photons, considered as a function of the excitation frequency $\Omega_{0}$. Consequently, this spectrum is determined by the formulae (21)-(23) for the time-dependent RSR spectrum within the limit $\eta \rightarrow \infty$. It is easy to show that it coincides with formula (12) for the luminescence spectrum if $\Omega$ and $\eta$ are substituted by $\Omega_{0}$ and $\eta_{0}$, respectively. Thus, in the time-dependent absorption spectrum also the compensation effect takes place, whereas, by selecting $\eta_{0}$, one can compensate $\gamma$ and $\eta_{0}$ (but not $\gamma_{1}$ ).

\section{Resonant absorber}

In the Mössbauer effect, instead of the spectrometer a resonant absorber (scatterer) is used. In this case, within the limits of a thin absorber $\left.{ }^{1}{ }^{1}\right]$

$$
C(\omega)=1-i C_{0}(\Omega-\omega+i \eta)^{-1},
$$

where $C_{0}(\Omega-\omega+i \eta)^{-1}$ is the resonant part of the complex dielectric constant. If the radiation described by formula (9) is passed through such filter, then the time-dependent spectrum of the transmitted emission is of the form 


$$
I \sim e^{-2 \gamma t}-\frac{2 C_{0} e^{-(\gamma+\eta) t}}{x^{2}+\bar{\gamma}^{2}}\left[x \sin x t-\bar{\gamma}\left(\cos x t-e^{-\bar{\gamma} t}\right)\right] .
$$

In this spectrum, also, the compensation of $\eta$ and $\gamma$ takes place: if $\eta=\gamma$, then $I \sim 1-2 C_{0} x^{-1} \sin x t$. This formula coinciding with the analogous formulae of investigations $[1,2,18]$ within the limits of a thin absorber, describes the minimum with the uncertainty-principle-limited halfwidth $t^{-1}$.

\section{Concluding remarks}

In this paper, the effect of the resolution $(\eta)$ of the spectral device on the width and shape of lines in time-dependent spectra has been considered. This effect was shown to differ essentially from the one in stationary spectra: if in the latter ones the narrowest lines are obtained at $\eta \rightarrow 0$, then in the former ones, they occur at $\eta>0$. In particular, in the time-dependent luminescence spectrum the lines with uncertaintyprinciple-limited halfwidth $t^{-1}$ can be obtained at a $\eta$ equal to the natural width $\gamma$, in the scattering spectrum, at a $\eta$ equal to the halfwidth of the excitation spectrum, $\eta_{0}$, and in the absorption spectrum, at $\gamma \Rightarrow \eta_{0}$. The reason of such an «unusual» effect of spectral resolution on a timedependent spectrum lies in the interference of signals, with a vatied delay (within the limits $\eta_{(0)}^{-1}$ ) which arises on the filtration of the finiteduration pulse by the spectrometer. It is this interference that leads to the compensation of $\eta$ and $\gamma$ in the luminescence spectrum, of $\eta$ and $\eta_{0}$ in the scattering spectrum, and of $\eta_{0}$ and $\gamma$ in the absorption spectrum.

In this work we suppose that the emission is first passed through the spectrometer and then registered at a certain (to be more exact, in a small interval $t, t+\delta t)$ time moment. Thus, first an analysis of the radiation over the spectrum is made, and then the time is measured. In this scheme, the result of the measurement essentially depends on the spectrometer resolution $\eta$, which affects the temporal properties of the spectrometer-transmitted radiation.

However, other schemes for measuring the time-dependent spectrum are also possible. For example, first the time is fixed (more exactly, the time interval $t, t+\tau)$ and then the spectrum is measured. To effectuate this scheme, evidently a time-lock before the spectrometer must be used (not after it, as it was in the preceding scheme). Here the spectrometer works like in a stationary case. Therefore, with regard to obtaining narrow lines, small $\eta$ are the best. A time-dependent spectrum of such type is described by formulae (2) and (3) if $C(t)$ is understood as the time-lock transmission. It is easy to show [19] that in this scheme the reverse time of the time-lock transmission $\tau^{-1}$ has the same role as the spectral resolution $\eta$ had in the first scheme. In particular, here also exists the compensation effect: by selecting $\tau^{-1}=\gamma$, the broadenings $\tau^{-1}$ and $\gamma$ can be compensated (but phase-relaxational broadening $\gamma_{1}$ cannot). Moreover, the lines with an uncertainty-principle-limited halfwidth can be obtained only if $\tau^{-1}$ is properly chosen.

In this study, the exponential transmission function (i.e. the Lorentz filtration function) was regarded. It can be shown, however, that the compensation effect, though incomplete, takes place for other transmission functions as well.

Thus, the nonmonotonic dependence of the width and shape of the line on the spectrometer resolution $\eta$ (or on the time-lock transmission time $\tau$ ) is a common feature of time-dependent spectra.

The authors are indebted to K. K. Rebane for discussions. 


$$
\begin{aligned}
I \sim & e^{-2 \eta_{0} t} k_{1} k_{3}\left|\alpha_{1} \delta_{3}\right|^{2}+e^{-2 n t}\left\{\left(\left|\alpha_{1}\right|^{2}+k_{2} k_{4}\left|\alpha_{3}\right|^{2}\right)\left|\delta_{1}\right|^{2}+\right. \\
& +\left(1-k_{1}\right)\left|\alpha_{2}\right|^{2}\left[\left(1-k_{2}\right)\left|\delta_{2}\right|^{2} e^{-2 \gamma^{t}}-2 \operatorname{Re} \delta_{1} \delta_{2}^{*} e^{-t / \delta_{1}}\right]+ \\
& +\left|\alpha_{1} \beta\left(1-e^{t / \beta}\right)\right|^{2}+2 \operatorname{Re}\left[\left(1-e^{t / \beta^{*}}\right)\left(\left|\alpha_{1}\right|^{2}-\alpha_{1} \alpha_{2}^{*} e^{-t / \delta_{1}}\right) \delta_{1} \beta^{*}\right]+ \\
& \left.+2 \operatorname{Re} \delta_{1} e^{-t / \delta_{1}}\left[\left|\alpha_{1}\right|^{2}\left(k_{1} \delta_{3}-2 \gamma_{1} \alpha_{2} \xi\right)+2 \gamma_{1} \alpha_{1} \alpha_{2}^{*} \alpha_{3} \xi^{*} e^{t / \beta^{*}}\right]\right\},
\end{aligned}
$$

where

$$
\begin{aligned}
& \alpha_{1}=\left(i x_{0}+\gamma_{0}+\gamma_{1}\right)^{-1}, \\
& \alpha_{2}=\left(i x_{0}+\gamma_{0}-\gamma_{1}\right)^{-1}, \\
& \alpha_{3}=\left(i x_{0}+\gamma_{0}+\gamma_{1}-2 \bar{\eta}\right)^{-1}, \\
& \beta=(i y+\bar{\eta})^{-1}, \\
& \delta_{1}=\left(i x+\bar{\gamma}+\gamma_{1}\right)^{-1}, \\
& \delta_{2}=\left(i x+\bar{\gamma}-\gamma_{1}\right)^{-1}, \\
& \delta_{3}=\left(i x+\bar{\gamma}+\gamma_{1}+2 \bar{\eta}\right)^{-1}, \\
& \xi=\left[i\left(x_{0}+x\right)+\bar{\eta}\right]^{-1}, \\
& k_{1}=\gamma_{1} / \gamma_{0}, \\
& k_{2}=\gamma_{1} / \bar{\gamma} \\
& k_{3}=\left(1+\frac{\gamma_{0}+\gamma_{1}}{\bar{\eta}}\right), \\
& k_{4}=\left(1-\frac{\bar{\gamma}+\gamma_{1}}{\bar{\eta}}\right) .
\end{aligned}
$$

\section{REFERENCES}

1. Lynch, F. J., Holland, R. E., Hamermesh, M. Phys. Rev., 120, № 2, 513-520 (1960).

2. Harris, S. M. Phys. Rev., 124, № 4, 1178-1185 (1961).

3. Triftshäuser, W., Craig, P. P. Phys. Rev. Lett., 162, № 2, 274-285 (1967).

4. Kobayashi, T., Shimizu, S. Phys. Lett., 54A, № 4, 311-312 (1975).

5. Koch, R., Realo, E. JETP Lett., 30, № 11, 679-682 (1979).

6. Koch, R., Realo, E. ENSV TA Toim. Füüs. Matem., 28, № 4, 374-377 (1979); 30, № $2, \quad 171-174$ (1981).

7. Rebane, K. K. J. Lumin., № 18/19, 693-701 (1979).

8. Hizhnyakov, V. V., Rebane, I. K. JETP, 74, № 3, 885-896 (1978).

9. Rebane, I. K., Tuul, A. L., Hizhnyakov, V. V. JETP, 77, № 4(10), 1302-1312 (1979).

10. Shimizu, F., Umezu, K., Takuma, H. Phys. Rev. Lett., 47, № 12, 825-827 (1981);

Shimizu, F., Shimizu, K., Takuma, H. Phys. Rev. A28, № 4, 2248-2253 (1983).

11. Zinth, W.. Nuss, M. C., Kaiser, W. Opt. Commun., 44, № 4, 262-266 (1983).

12. Coleman, P. E., Kagan, D., Knight, P. L. Opt. Commun., 36, № 2, 127-130 (1981).

13. Eberly, J. H., Kunasz, C. V., Wodkiewicz K. J. Phys., B13, 217-239 (1980).

14. Glauber, R. J. Phys. Rev., 130, № 6, 2529-2539 (1963).

15. Eberly, J. H., Wodkiewicz, K. J. Opt. Soc. Amer., 67, № 9, 1252-1261 (1977)

16. Saari, P., Aaviksoo, J., Freiberg, A., Timpmann, K. Opt. Commun., 39, № 1/2, 94-98 (1981).

17. Born, H., Wolf, E. Principles of Optics (5th ed.). Oxford, Pergamon, 1975, Sec. 7.6.

18. Wu, C. S., Lee, Y. K., Benzcer-Koller, N., Simms, P. Phys. Rev. Lett., 5, № 9, $432-435$ (1960).

19. Rebane, I., Hizhnyakov, V. ENSV TA Toim. Füüs. Matem., 30, № 1, 1-8 (1981). 


\section{JOONTE KITSENEMINE AJAST SOLTUVATES SPEKTRITES}

Arvestades homogeenset (modulatsioonilist) ja mittehomogeenset laienemist, vaadeldakse ajast sōltuvaid resonantseid neeldumise, luminestsentsi ja sekundaarse kiirguse spektreid. On näidatud, et kitsaimad spektrijooned saadakse mitte spektraalse lahutuse $\eta=0$ juhul, nagu see on statsionaarsete spektrite korral, vaid sobivalt valitud $\eta>0$ juhul. Sellisel puhul võib aja kasvades joon minna kitsamaks kui $\eta$.

\section{В. ХИЖНЯКОВ, Инна РЕБАНЕ}

\section{СУЖЕНИЕ ЛИНИИ В ЗАВИСЯЩИХ ОТ ВРЕМЕНИ СПЕКТРАХ}

Рассматриваются зависящие от времени спектры резонансного вторичного свечения, поглощения и люминесценции с учетом однородного (модуляционного) и неоднородного уширений. Показано, что в пределах малого спектрального разрешения $\eta$ ширина линии уменьшается с ростом $\eta$. Линии с полушириной, определенной по принципу соотношения неопределенностей, могут быть получены только при подходящем подборе $\eta>0$ и при малом модуляционном уширении. В этом случае с возрастанием времени линии сужаются до ширин, меньших чем $\eta$. 